Algebraic $8 \mathcal{G}$ Geometric $\mathcal{T}$ opology

Volume 5 (2005) 741-750

Published: 23 July 2005

ATG

\title{
Boundary slopes (nearly) bound cyclic slopes
}

\author{
Thomas W. Mattman
}

\begin{abstract}
Let $r_{m}$ and $r_{M}$ be the least and greatest finite boundary slopes of a hyperbolic knot $K$ in $S^{3}$. We show that any cyclic surgery slopes of $K$ must lie in the interval $\left(r_{m}-1 / 2, r_{M}+1 / 2\right)$.
\end{abstract}

AMS Classification 57M25; 57M27

Keywords Dehn surgery, character variety, exceptional surgery, boundary slope

\section{Introduction}

In [M1, M2] we observed that the Seifert surgeries of $(-3,3, n)$ pretzel knots follow an interesting pattern, summarised in the table below. For each positive integer $n$, the Seifert surgeries of the $(-3,3, n)$ pretzel lie between the boundary slopes 0 and $8 /(n+1)$. Indeed, all integral slopes in the interval $(0,8 /(n+1))$ are Seifert.

\begin{tabular}{|c|c|c|c|c|c|c|c|}
\hline$n$ & 1 & 2 & 3 & 4 & 5 & 6 & $\geq 7$ \\
\hline $8 /(n+1)$ & 4 & $8 / 3$ & 2 & $8 / 5$ & $4 / 3$ & $8 / 7$ & $\leq 1$ \\
\hline Seifert Surgeries & $1,2,3$ & 1,2 & 1 & 1 & 1 & 1 & none \\
\hline
\end{tabular}

Some other famous knots also share this pattern; all integral slopes between two boundary slopes (shown in bold typeface) are Seifert:

\begin{tabular}{|l|c|}
\hline Knot & Non-trivial exceptional surgeries \\
\hline Figure 8 & $-\mathbf{4},-3,-2,-1, \mathbf{0}, 1,2,3, \mathbf{4}$ \\
$(-2,3,7)$ & $\mathbf{1 6}, 17,18, \mathbf{3 7} / \mathbf{2}, 19, \mathbf{2 0}$ \\
Twist Knots & $\mathbf{0}, 1,2,3, \mathbf{4}$ \\
\hline
\end{tabular}

Based on this and other evidence, Kimihiko Motegi posed the question: "Are Seifert surgeries bounded by boundary slopes?" In other words, if slope $t$ is a Seifert surgery slope, are there necessarily boundary slopes $r_{m}$ and $r_{M}$ with 
$r_{m} \leq t \leq r_{M}$ ? In [IMS, we construct a parameter $c$, such that $r_{m}-c \leq t \leq$ $r_{M}+c$. More precisely, we can reformulate [MS, Corollary 3] as follows. (In part 3 of Theorem 1 the constant $s$ is the minimal Culler-Shalen norm defined in Section 2 below while $A$ counts the characters of non-abelian representations of the knot exterior that factor through the surgery. We refer the reader to [MS for details.)

Theorem 1 (Corollary 3 of [MS]) Let $r_{m}$ and $r_{M}$ be the least and greatest finite boundary slopes of a hyperbolic knot $K$ and $t$ a non-trivial exceptional surgery slope. Then $r_{m}-c \leq t \leq r_{M}+c$ where $c$ depends on the type of slope $t$.

(1) If $t$ is cyclic, $c=1$.

(2) If $t=a / b$ is finite, $c=3 / b$.

(3) If $t=a / b$ is a Seifert fibred slope, $c=(1+2 A / s) / b$.

In this formulation, Motegi's conjecture corresponds to showing $c=0$ for a Seifert fibred surgery. That $c=1$ for a cyclic surgery was first shown by Dunfield $\mathrm{Du}$. In the current article, we show that for a cyclic surgery, we can take $c=1 / 2$.

Theorem 2 If $t$ is a non-trivial cyclic surgery on a hyperbolic knot $K$ in $S^{3}$ and $r_{m}$ and $r_{M}$ are the least and greatest finite boundary slopes of $K$, then $r_{m}-\frac{1}{2}<t<r_{M}+\frac{1}{2}$.

Moreover, the theorem applies more generally to a hyperbolic knot in a manifold with cyclic fundamental group whose exterior satisfies $H^{1}\left(M, \mathbb{Z}_{2}\right)=\mathbb{Z}_{2}$.

In light of Theorem 1] it is natural to extend Motegi's question about Seifert surgeries to exceptional surgeries in general:

Question 1 Do boundary slopes bound exceptional slopes?

In other words, for a hyperbolic knot $K$ in $S^{3}$ do all non-trivial exceptional surgery slopes lie in the interval $\left[r_{m}, r_{M}\right]$ between the least and greatest finite boundary slopes? Note that, by the Cabling Conjecture, $K$ should have no reducible surgeries and that toroidal surgeries are themselves boundary slopes and will, therefore, necessarily lie in $\left[r_{m}, r_{M}\right]$. The real question is whether other types of exceptional surgeries (i.e., cyclic, finite, Seifert fibred) must also lie in this interval. It is the Seifert case that gives the largest values for $c$ 
in Theorem 1 and, in any case, cyclic and finite surgeries are thought to be examples of Seifert surgeries. Thus, an affirmative answer to Motegi's question is likely to imply the same for all types of exceptional surgeries.

In Section 2 we provide definitions and discuss the geometry of the Culler-Shalen norm. In Section 3, we prove Theorem 2 .

\section{Definition, geometry of the Culler-Shalen norm}

Let $K$ be a hyperbolic knot in $S^{3}$ and let $M=S^{3} \backslash N(K)$ denote the knot exterior. Fixing the usual meridian, longitude basis $\{\mu, \lambda\}$, the element $\gamma=$ $a \mu+b \lambda$ of $H_{1}(\partial M ; \mathbb{Z})$ will be represented as $(a, b)$. This class can be identified with the "slope" $r_{\gamma}=a / b$ in $\mathbb{Q} \cup\left\{\frac{1}{0}\right\}$. We will occasionally wish to change our framing which amounts to replacing $\lambda$ by $k \mu+\lambda$ and to changing coordinates by $(a, b) \mapsto(a-b k, b)$.

Let $M(r)$ denote the manifold obtained by Dehn surgery along slope $r$ (i.e., $M(r)$ is constructed by attaching a solid torus to $M$ such that the boundaries of meridional disks are curves of slope $r$ in $\partial M)$. We will call $r$ a cyclic (respectively finite) slope if $\pi_{1}(M(r))$ is cyclic (resp. finite). If $M(r)$ admits the structure of a Seifert fibred space, we call $r$ a Seifert fibred slope. Since $M\left(\frac{1}{0}\right)=S^{3}$, we refer to meridional surgery along slope $r_{\mu}=\frac{1}{0}$ as trivial surgery.

If there is an essential surface $\Sigma$ in $M$ that meets $\partial M$ in a non-empty set of parallel curves of slope $r$, we call $r$ a boundary slope. If there is such a $\Sigma$ that is not a fibre in a fibration of $M$ over $S^{1}, r$ is a strict boundary slope. For example, by applying the loop theorem to a Seifert surface of $K$, we observe that 0 is a boundary slope. We will say $r$ is a finite boundary slope if it is a boundary slope and $r \neq \frac{1}{0}$.

The proof of Theorem 2 depends on the geometry of the Culler-Shalen norm of $K$. We introduce some of the main properties of this norm and refer the reader to CGLS, Chapter 1] for a more complete account.

Let $R=\operatorname{Hom}\left(\pi, \mathrm{SL}_{2}(\mathbb{C})\right)$ denote the set of $\mathrm{SL}_{2}(\mathbb{C})$-representations of the fundamental group $\pi$ of $M$. Then $R$ is an affine algebraic set, as is $X$, the set of characters of representations in $R$.

For $\gamma \in \pi$, define the regular function $I_{\gamma}: X \rightarrow \mathbb{C}$ by $I_{\gamma}\left(\chi_{\rho}\right)=\chi_{\rho}(\gamma)=$ $\operatorname{trace}(\rho(\gamma))$. By the Hurewicz isomorphism, a class $\gamma \in L=H_{1}(\partial M, \mathbb{Z})$ determines an element of $\pi_{1}(\partial M)$, and therefore an element of $\pi$ well-defined up to conjugacy. A norm curve is a one-dimensional irreducible component of $X$ on 
which no $I_{\gamma}(\gamma \in L \backslash\{0\})$ is constant. For example, the irreducible component, $X_{0}$, that contains the character of the holonomy representation is a norm curve.

The terminology reflects the fact that we may associate to $X_{0}$ a norm $\|\cdot\|$ on $H_{1}(\partial M, \mathbb{R})$ called a Culler-Shalen norm in the following manner. Let $\widetilde{X}_{0}$ be the smooth projective model of $X_{0}$ which is birationally equivalent to $X_{0}$. The birational map is regular at all but a finite number of points of $\widetilde{X}_{0}$ which are called ideal points of $\widetilde{X}_{0}$. The function $f_{\gamma}=I_{\gamma}^{2}-4$ is again regular and so can be pulled back to $\widetilde{X}_{0}$. For $\gamma \in L,\|\gamma\|$ is the degree of $f_{\gamma}: \widetilde{X}_{0} \rightarrow \mathbb{C P}^{1}$. The norm is extended to $H_{1}(\partial M, \mathbb{R})$ by linearity.

Let $s=\min _{0 \neq \gamma \in H_{1}(\partial M, \mathbb{Z})}\|\gamma\|$ denote the minimal norm. The norm disc of radius $s$ is a convex, finite-sided polygon $P$ that is symmetric about the origin. We will call $P$ the fundamental polygon. The ideal points of $\widetilde{X}_{0}$ can be associated with a set $\mathcal{B}$ of strict boundary slopes of the knot and the vertices of $P$ occur at rational multiples of the classes of slopes in $\mathcal{B}$. It follows that $\mathcal{B}$ must contain at least two slopes. One of the main results of [CGLS] is that if $r_{\gamma}$ is a cyclic slope that is not a strict boundary slope then $\|\gamma\|=s$. Moreover, $r_{\gamma}$ is either integral or trivial (i.e., $r_{\gamma}=\frac{1}{0}$ ).

\section{Proof of Theorem 2}

We will prove two propositions before coming to the proof of the theorem. The main external inputs for our argument are the Cyclic Surgery Theorem CGLS and Theorems 4.1 and 4.2 of $[\mathrm{Du}$. Thus, although we formulate our results in terms of a knot in $S^{3}$, they carry over to the case of a hyperbolic knot in a manifold with cyclic fundamental group whose exterior satisfies $H^{1}\left(M, \mathbb{Z}_{2}\right)=$ $\mathbb{Z}_{2}$.

Proposition 1 Let $K$ be a hyperbolic knot in $S^{3}$. Let $r_{M}$ be the greatest finite boundary slope of $K$. Suppose $\gamma$ is a non-trivial cyclic class with $r_{\gamma}=$ $n>0$. Then $n \leq r_{M}+\frac{1}{2}$.

Remark In fact, we will show that $n \leq r+\frac{1}{2}$ where $r$ is the greatest finite boundary slope associated to the norm curve $X_{0}$. In particular, $r$ is a strict boundary slope.

Proof Let $X_{0}$ be the norm curve that contains the character of the holonomy representation and let $\mathcal{B}$ be the associated set of boundary slopes. If $r_{\gamma} \in \mathcal{B}$, then $n=r_{\gamma} \leq r_{M}$. So we may assume $r_{\gamma} \notin \mathcal{B}$. 
Suppose, for a contradiction, that $n>r_{M}+\frac{1}{2}$. Without loss of generality, we may assume $r_{M} \in \mathcal{B}$ (otherwise replace $r_{M}$ by the greatest finite boundary slope in $\mathcal{B}$ ). Our goal is to argue that $(n-1,1)$ is in the interior of $P$ (see Figure 1) where $P$ is the fundamental polygon of the Culler-Shalen norm $\|\cdot\|$

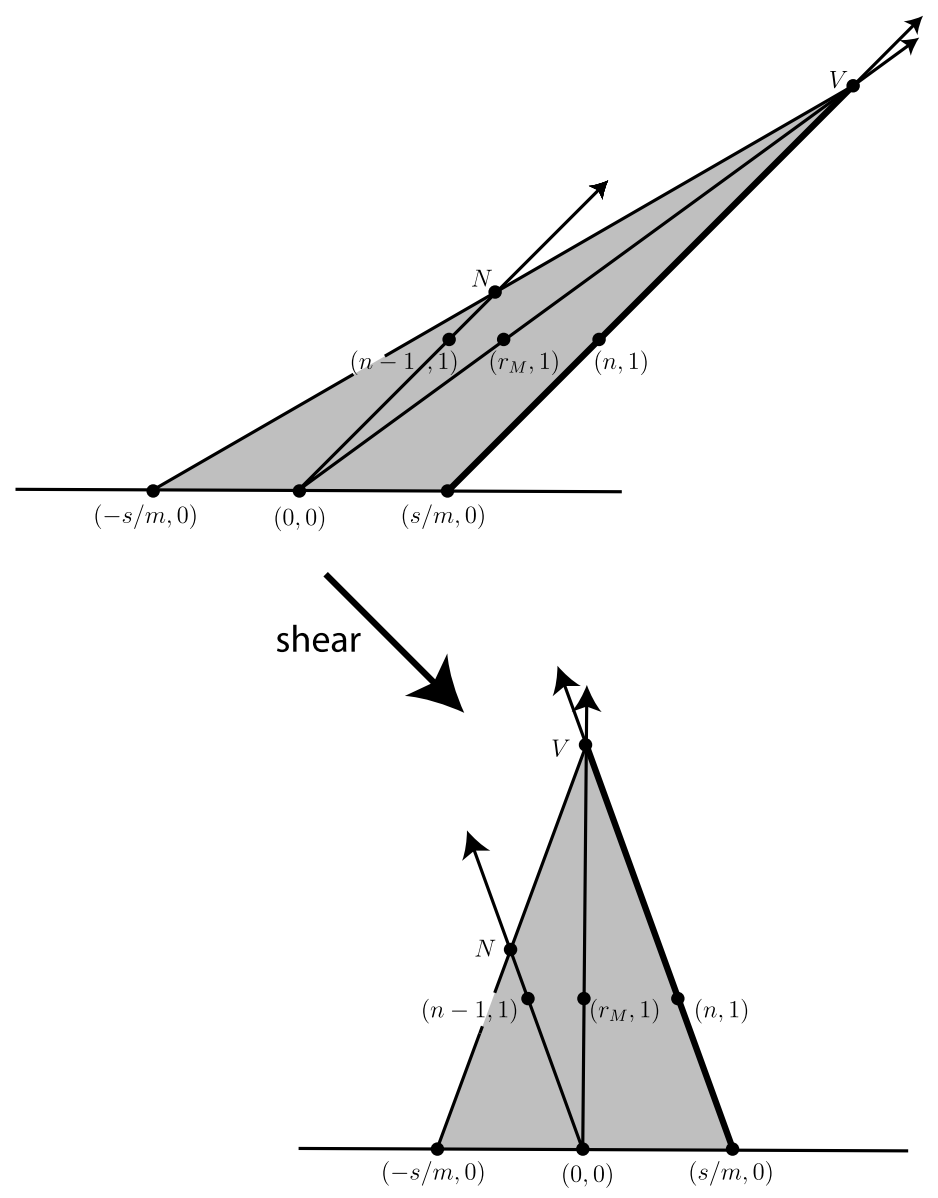

Figure 1: The geometry of $P$ assuming $n>r_{M}+\frac{1}{2}$

associated to $X_{0}$. Let us outline the argument. First, it has already been shown that $r_{M}$ must be non-integral $[\mathrm{Du}$. Construct the line from $(s / m, 0)$ through $(n, 1)$, where $m=\|\mu\|$ and $s$ is the minimal norm. This line will form part of the boundary of $P$ and continues to the vertex $V$ determined by the boundary slope $r_{M}$. By convexity, the line joining $(-s / m, 0)$ to $V$ is also in $P$. It follows that $(n-1,1)$ is in the interior of $P$ so that $\|(n-1,1)\|<s$. (This is "obvious" if one applies a shear as in Figure 1) This is a contradiction as $s$ is defined to 
be the minimal norm. Thus, we conclude $n \leq r_{M}+\frac{1}{2}$.

Now let's fill in the details. Since $n>r_{M}+\frac{1}{2}$, if $r_{M}$ is an integer, then $r_{M} \leq n-1$. However, by $\mathrm{Du}$, Theorem 4.2] there is a strict boundary slope $r_{\delta} \in \mathcal{B}$ with $\left|n-r_{\delta}\right|<1$. Then $r_{M}<r_{\delta}$ in contradiction to the choice of $r_{M}$. Therefore, $r_{M}$ is not an integer. Moreover, since there is a strict boundary slope $\delta$ with $\left|n-r_{\delta}\right|<1$ we have $n-1<r_{M}<n$.

We next construct the vertex $V$ of $P$ corresponding to the boundary slope $r_{M}$. Let $\|\mu\|=m$. (If $r_{\mu}=\frac{1}{0} \notin \mathcal{B}$, then $m=s$.) The point $(s / m, 0)$ is then in $\partial P$. Since $r_{M}$ is maximal among slopes in $\mathcal{B}$, and $r_{M}<n<\infty$, the segment joining $(s / m, 0)$ and $(n, 1)$ is part of the boundary of $P$. This segment has equation $y=(x-s / m) /(n-s / m)$. It continues to the line $y=x / r_{M}$. (Since there are no strict boundary slopes between $n$ and $r_{M}$, the segment has no vertex before it reaches the line $y=x / r_{M}$ corresponding to the boundary slope $r_{M}$.) These lines meet at the point

$$
V=\frac{s / m}{r_{M}+s / m-n}\left(r_{M}, 1\right)
$$

which is therefore a vertex of $P$.

Since $P$ is convex, the segment joining $V$ and $(-s / m, 0)$ (both in $P$ ) is contained in $P$. We argue that the point $N$ where this segment crosses $y=$ $x /(n-1)$ is above the line $y=1$. Indeed, the segment has the equation

$$
y=\frac{x+s / m}{2 r_{M}+s / m-n} .
$$

It meets the line $y=x /(n-1)$ at the point

$$
N=\frac{s / m}{2\left(r_{M}-n\right)+1+s / m}(n-1,1)
$$

which is therefore in $P$. Let $y_{N}$ denote the $y$ coordinate of $N$.

$$
\begin{aligned}
n>r_{M}+\frac{1}{2} & \Rightarrow 0>2\left(r_{M}-n\right)+1 \\
& \Rightarrow s / m+2\left(r_{M}-n\right)+1<s / m \\
& \Rightarrow y_{N}=\frac{s / m}{2\left(r_{M}-n\right)+1+s / m}>1
\end{aligned}
$$

Since $y_{N}>1$, the point $(n-1,1)$ is in the interior of $P$ and, therefore, $\|(n-1,1)\|<s$. This is a contradiction as $s$ is defined to be the minimal norm. We conclude that $n \leq r_{M}+\frac{1}{2}$. 
We will now show that Proposition 1 can be strengthened to a strict inequality if the meridian is not a strict boundary class for the norm curve $X_{0}$. The argument makes use of the idea of the diameter $D$ of the set of boundary slopes. Culler and Shalen [CS] showed that $D \geq 2$. This inequality is sharp by an example of Dunfield of a knot in a manifold with cyclic fundamental group (see [CS]). For hyperbolic knots in $S^{3}$, the smallest known diameter is $D=8$ for the Figure 8 knot.

Proposition 2 Let $X_{0}$ be the norm curve containing the character of the holonomy representation for the hyperbolic knot $K \in S^{3}$. Let $\mathcal{B}$ be the associated boundary slopes and suppose that $r_{\mu}=\frac{1}{0} \notin \mathcal{B}$. Let $\gamma$ be a non-trivial cyclic class with $r_{\gamma}=n>0$. Let $r_{m}$ and $r_{M}$ denote the least and greatest boundary slopes in $\mathcal{B}$. Let $D=\operatorname{Diam}(\mathcal{B})=r_{M}-r_{m}$. Then $n \leq r_{M}+1-\frac{1}{2}(D-\sqrt{D(D-2)})<r_{M}+\frac{1}{2}$.

Remark The difference between $n$ and $r_{M}$ goes to zero as $D$ approaches 2 . For $D=8$ (Figure 8 knot) we have $1-\frac{1}{2}(D-\sqrt{D(D-2)})=-3+2 \sqrt{3} \approx 0.46$.

Proof Now $\|\mu\|=s$ and $\pm(1,0) \in \partial P$. If $n \leq r_{M}$, the proposition holds. So, using Proposition 1, we'll assume $r_{M}<n \leq r_{M}+\frac{1}{2}$. Let's change the framing so that $n$ becomes 1 . We will use $\sim$ to refer to measurements in the new framing. Thus, $\tilde{n}=1, \tilde{r}_{M}=r_{M}-n+1$ and $\tilde{r}_{m}=r_{m}-n+1$. The line through $(\tilde{n}, 1)$ and $(1,0)$ is then vertical and and meets $y=x / \tilde{r}_{M}$ at $V=\left(1,1 / \tilde{r}_{M}\right)$ (see Figure 2).

The segment in $\partial P$ which passes through $V,(\tilde{n}, 1)$, and $(1,0)$ continues to the line $y=x / \tilde{r}_{m}$ as there are no boundary slopes between $\tilde{r}_{m}$ and $\tilde{r}_{M}$ to provide a vertex. The intersection point $W^{\prime}=\left(1,1 / \tilde{r}_{m}\right)$ is therefore a vertex of $P$ as is its reflection $W=-W^{\prime}$.

Since $P$ is convex, the segment joining $V$ and $W$ is contained in $P$. It meets the line $y=0$ at the point

$$
M=\left(0, \frac{\tilde{r}_{m}-\tilde{r}_{M}}{2 \tilde{r}_{M} \tilde{r}_{m}}\right)
$$

Since $\|(0,1)\| \geq s$, the $y$ coordinate of $M$ cannot exceed 1 (note that $D=$ $\left.\tilde{r}_{M}-\tilde{r}_{m}\right)$ :

$$
1 \geq \frac{D}{2 \tilde{r}_{M}\left(D-\tilde{r}_{M}\right)} .
$$




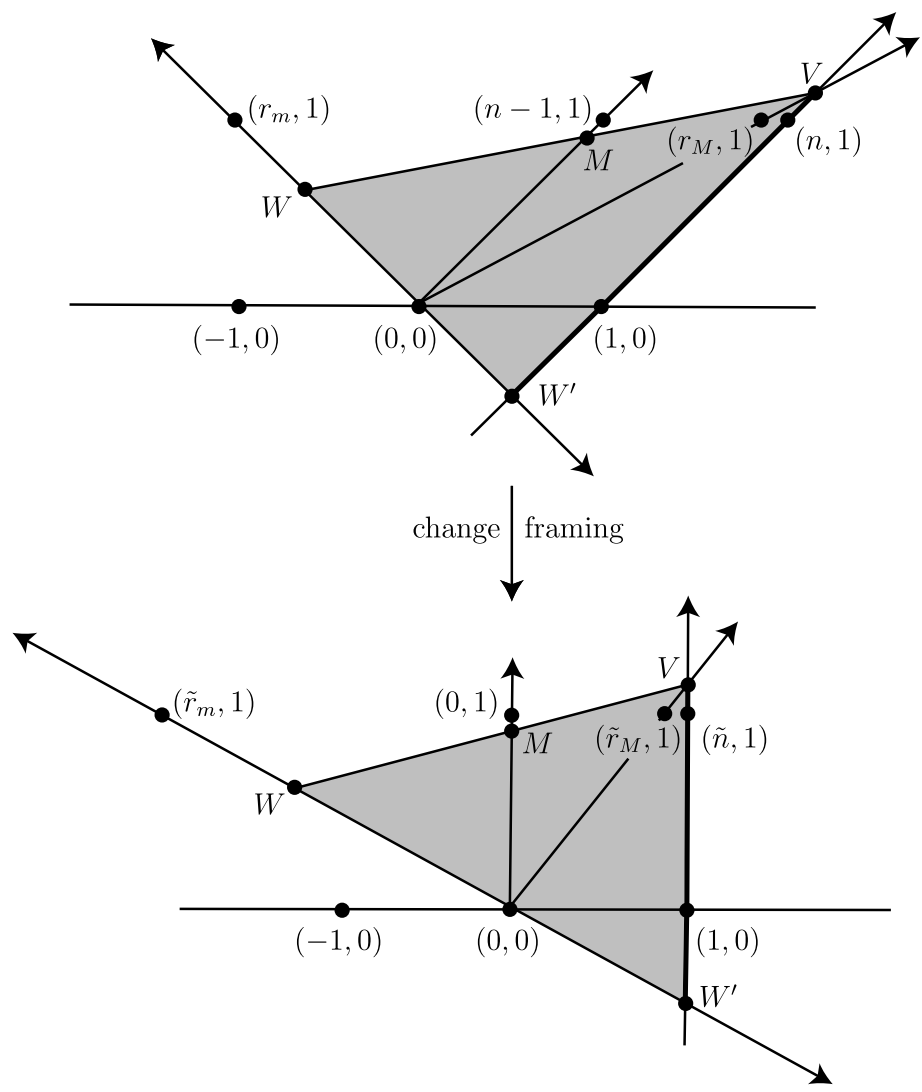

Figure 2: The geometry of $P$ assuming $\|\mu\|=s$

By the previous proposition, $\tilde{r}_{M} \geq \frac{1}{2}$. Since $n \geq r_{M}$, we have $\tilde{r}_{M} \leq 1$. Recall CS that $D \geq 2$.

$$
\begin{aligned}
1 \geq \frac{D}{2 \tilde{r}_{M}\left(D-\tilde{r}_{M}\right)} & \Rightarrow 2 \tilde{r}_{M}\left(D-\tilde{r}_{M}\right) \geq D \\
& \Rightarrow 2 \tilde{r}_{M}^{2}-2 D \tilde{r}_{M}+D \leq 0 \\
& \Rightarrow \quad \tilde{r}_{M} \geq \frac{1}{2}(D-\sqrt{D(D-2)})
\end{aligned}
$$

Since $\tilde{r}_{M}=r_{M}-n+1$, we have, $n \leq r_{M}+1-\frac{1}{2}(D-\sqrt{D(D-2)})$, as required.

We are now in a position to prove Theorem 2 . 
Let $K$ be a hyperbolic knot in $S^{3}$ and let $r_{\gamma}=n$ be a non-trivial cyclic surgery slope. Then, by the Cyclic Surgery Theorem [CGLS, $n$ is an integer and, without loss of generality, we may assume $n \geq 0$. Since 0 is a boundary slope, $r_{m} \leq 0$ so that $r_{m}-\frac{1}{2}<n$. Similarly, if $n=0, r_{M}+\frac{1}{2}>n$. Thus, in order to prove Theorem [2 it is enough to show $n<r_{M}+\frac{1}{2}$ when $0<n=r_{\gamma}$ is a cyclic surgery slope.

By Proposition 1, $n \leq r_{M}+\frac{1}{2}$, so it remains only to show that equality is not possible. Suppose then (for a contradiction) that $n=r_{M}+\frac{1}{2}$ and change the framing so that $n$ goes to $\tilde{n}=1$. Then $\tilde{r}_{M}=\frac{1}{2}$. Following the argument in the proof of Proposition 1, the line from the point $E=(s / m, 0)$ through $V$ is part of the boundary of $P$. Since $m=\|\mu\|, m$ is at least as big as the minimal norm $s$. Thus, $E$ lies on the half open segment $((0,0),(1,0)]$. Similarly, $\|(1,2)\| \geq s$ and, therefore, $V$, which lies on the boundary of $P$, must be in the half open segment $((0,0),(1,2)]$. However, the line through $E$ and $V$ also passes through $(\tilde{n}, 1)=(1,1)$. The only consistent way to account for all these facts is to have $V=(1,2)$ and $E=(1,0)$. In other words, $\|\mu\|=s$. Reviewing the argument of Proposition 2, we see that $\|\mu\|=s$ is exactly the extra input needed to deduce that $n<r_{M}+\frac{1}{2}$. Thus, we conclude that $n<r_{M}+\frac{1}{2}$. This is absurd since we began by assuming $n=r_{M}+\frac{1}{2}$. The contradiction shows that, in fact, equality is not possible in Proposition 1. This completes the proof of Theorem 2 ,

Acknowledgments I am grateful to Kimihiko Motegi for suggesting the question that motivated this study and to Masaharu Ishikawa and Koya Shimokawa for helpful conversations. I thank the referee for many suggestions that significantly improved this paper.

\section{References}

[CGLS] M Culler, C McA Gordon, J Luecke, P B Shalen, Dehn surgery on knots, Ann. of Math. 125 (1987) 237-300 MathReview

[CS] M Culler, P B Shalen, Boundary slopes of knots, Comment. Math. Helv. 74 (1999) 530-547 MathReview

[Du] N Dunfield, Cyclic surgery, degrees of maps of character curves, and volume rigidity for hyperbolic manifolds, Invent. Math. 136 (1999) 623-657 MathReview

[IMS] M Ishikawa, T W Mattman, K Shimokawa, Exceptional surgery and boundary slopes, (submitted) arXiv:math.GT/0211147 
[M1] T W Mattman, The Culler-Shalen seminorms of pretzel knots, Ph.D. Thesis, McGill University, Montreal (2000) available at:

http://www.csuchico.edu/math/mattman

[M2] T W Mattman, The Culler-Shalen seminorms of the (-3,3,4) pretzel knot, Proc. of Knot Theory - dedicated to Prof. Murasugi (Univ. of Toronto '99) (2000) 212-218 arXiv:math.GT/0102049

Department of Mathematics and Statistics, California State University, Chico Chico, CA95929-0525, USA

Email: TMattman@CSUChico.edu

Received: 16 November 2004 